\title{
Electrocardiography as the First Step for the Further Examination of Cardiac Involvement in Myasthenia Gravis
}

\author{
Takao Kato, ${ }^{1,2}$ Sayako Hirose, ${ }^{1}$ Shogo Kumagai, ${ }^{1}$ Akihiko Ozaki, ${ }^{3}$ \\ Sadayuki Matsumoto, ${ }^{3}$ and Moriaki Inoko ${ }^{1}$ \\ ${ }^{1}$ Cardiovascular Center, Tazuke Kofukai Medical Research Institute, Kitano Hospital, Osaka 530-8480, Japan \\ ${ }^{2}$ Department of Cardiovascular Medicine, Graduate School of Medicine, Kyoto University, Kyoto 606-8507, Japan \\ ${ }^{3}$ Department of Neurology, Tazuke Kofukai Medical Research Institute, Kitano Hospital, Osaka 530-8480, Japan \\ Correspondence should be addressed to Takao Kato; takao-kato@kitano-hp.or.jp
}

Received 21 October 2015; Revised 15 December 2015; Accepted 27 December 2015

Academic Editor: Natale Daniele Brunetti

Copyright (C) 2016 Takao Kato et al. This is an open access article distributed under the Creative Commons Attribution License, which permits unrestricted use, distribution, and reproduction in any medium, provided the original work is properly cited.

\begin{abstract}
Introduction. Cardiac involvement of myasthenia gravis (MG) accompanies a poor prognosis. In the present study, we aimed to investigate the relationship between ECG abnormality and cardiac involvement. Methods. Of 178 patients diagnosed with MG between 2001 and 2013 at our hospital, we retrospectively analyzed consecutive 58 patients who underwent both ECG and echocardiography and without underlying cardiovascular disease. ECG abnormalities were defined by computer-assigned Minnesota-codes. Cardiac damage was defined as either (1) ejection fraction (EF) $<55 \%$ on echocardiography or (2) elevated $E / e^{\prime}$, the ratio of mitral velocity to early diastolic velocity of the mitral annulus $>8$ on echocardiography. Results. Thirty-three patients (56.8\%) had ECG abnormality. An elevated $E / e^{\prime}$ was observed in patients with ECG abnormality compared to those without ECG abnormality (11.2 $\pm 3.2,8.7 \pm 2.2$, resp., $p=0.03)$. Among patients with ECG abnormality, 14 of 15 patients showed cardiac damage. Among patients without ECG abnormality, 6 of 33 patients showed cardiac damage $(p=0.003)$. Reduced EF was observed in five patients (8.6\%) with ECG abnormality and none in patients without ECG abnormality. Conclusions. ECG may aid as the first step for the further examination of cardiac damage in patients with MG.
\end{abstract}

\section{Introduction}

Myasthenia gravis (MG) primarily affects skeletal muscle via production of autoantibodies against nicotinic acetylcholine receptors in the motor endplates; heart muscle is a rare autoimmune target [1,2]. Patients with MG may show several kinds of cardiac involvement, ranging from asymptomatic changes on electrocardiography (ECG) to ventricular tachycardia, myocarditis, conduction disorder, heart failure, and sudden death [2-5]. Detection of cardiac involvement in MG is important because this cardiomyopathy may be treatable $[4,5]$. In the present study, we aimed to investigate the relationship between ECG abnormality and cardiac damage by echocardiography in order to aid in early detection and timely treatment.

\section{Methods}

Of 178 patients diagnosed with MG between 2001 and 2013 at our hospital, we retrospectively analyzed consecutive 58 patients who underwent both ECG and echocardiographic examinations and without underlying cardiovascular disease (ischemic heart disease, valvular heart disease, and hypertensive heart disease). ECG abnormalities were defined by standardized computer-assigned Minnesota-codes [6, 7]. Echocardiography was performed by cardiac sonographers according to the guidelines [8].

Cardiac damage was defined as either (1) ejection fraction (EF) $<55 \%$ on echocardiography (the EF of a normal heart may be between $55 \%$ and $70 \%$ ) according to the practice guideline $[8,9]$ or (2) elevated $E / e^{\prime}$, the ratio of mitral velocity 
TABLE 1: Characteristics of electrocardiographic abnormality.

\begin{tabular}{lcc}
\hline Characteristic & $n(\%)$ & $\begin{array}{c}\text { With left ventricle } \\
\text { damage, } n\end{array}$ \\
\hline $\begin{array}{l}\text { Persistent atrial } \\
\text { fibrillation }\end{array}$ & $6(10.3)$ & 3 \\
Atrioventricular block & $4(6.8)$ & 3 \\
ST depression & $17(29.3)$ & 9 \\
Negative T wave & $17(29.3)$ & 8 \\
\hline
\end{tabular}

to early diastolic velocity of the mitral annulus $\left(E / e^{\prime}\right)>8$ on echocardiography (the $E / e^{\prime}$ of a normal left ventricular enddiastolic pressure may be $<8)[8,10]$.

The study conformed to the ethical guidelines of the 1975 Declaration of Helsinki and was approved by the Institutional Review Board of Kitano Hospital. All data are expressed as mean \pm SD. Differences between groups were compared by using the Mann-Whitney $U$ test, while categorical variables were compared by using Pearson's chi-square test. In all tests, $p$ values of $<0.05$ were considered significant.

\section{Results}

Thirty-three patients (56.8\%) had ECG abnormality: 10.3\% had atrial fibrillation, $6.8 \%$ had atrioventricular block, $29.3 \%$ had nonspecific ST depression, and $29.3 \%$ had negative $\mathrm{T}$ wave (Table 1). There were no differences in comorbidities or clinical characteristics of MG patients with or without ECG abnormality (Table 2). On echocardiography, there was a difference in $E / e^{\prime}$ between patients with or without ECG abnormality $(11.2 \pm 3.2,8.7 \pm 2.2$, resp., $p=0.03$, Table 3$)$. Among patients with ECG abnormality, 14 of 15 patients showed the suggested cardiac damage. Among patients without ECG abnormality, 6 of 33 patients showed the suggested cardiac damage ( $p=0.003$, Table 4$)$. The sensitivity of ECG abnormality for detecting cardiac damage was 0.700 (95\% confidence interval [CI], 0.525-0.836), while its specificity was 0.711 (95\% CI, 0.618-0.782). The positive predictive value was 0.560 (95\% CI, 0.420-0.669), while the negative predictive value was 0.818 (95\% CI, 0.712-0.901).

When we limited the cases by reduced EF, five patients (8.6\%) had reduced EF (EFs of $10 \%, 26 \%, 43 \%, 49 \%$, and $54 \%$, resp.) with diffuse hypokinesis; all 5 had ECG abnormality with high $E / e^{\prime}$ values of $12 \pm 3.6$. Among patients without ECG abnormality, none of the patients showed reduced EF. Of the 5 patients with reduced EF, 1 patient with an EF of $30 \%$ died of colon cancer, and 4 patients were administered immunosuppressive therapy. Three of the 4 patients with EFs of $43 \%, 47 \%$, and $54 \%$, respectively, had a good clinical course, while 1 patient with an EF of $10 \%$ died of acute decompensated heart failure approximately 1 month after self-discontinuation of immunosuppressive therapy.

\section{Discussion}

Cardiac manifestation is thought to be relatively rare in MG. However, it has been reported that approximately $16 \%$ of patients with MG have various cardiac manifestations, such as arrhythmia and heart failure [1]. Autoimmunologic mechanisms are considered to be contributory to such cardiac involvement [2], such as the antibody for ryanodine receptor (RyR), the antibody for both beta 1 and beta 2 adrenergic receptors, the anti-titin antibodies, and the antibody for Kv1.4-a muscular voltage-gated potassium channel [2]. Arrhythmia is the most common clinical manifestation of unexplained cardiac disease in MG, occurring in as many as $8 \%$ of cases [1], which is consistent with the findings of the present study. The prevalence of abnormal ECG is also reported varying from $16 \%$ to $88 \%$ of patients with $\mathrm{MG}$ $[1,11,12]$, probably due to the inclusivity of MG patients who underwent ECG; however, no demonstration of any clinical significance was provided. This study validated the presence of ECG abnormality in patients with MG, including arrhythmia, conduction abnormality, and the abnormality of ST portion and $\mathrm{T}$ wave. We also showed that a small number of patients without ECG abnormality demonstrated cardiac damage, which comprised only diastolic impairment and did not include systolic impairment. These results implied the utility of ECG. If the abnormality exists, further examination by echocardiography may be necessary for the detection of systolic impairment in patients with MG, which indicates a poor prognosis but can be treated by immunosuppressant therapy $[1,2,4]$. Therefore, the presence or absence of ECG abnormality may be useful as the first step for the further examination of cardiac damage in patients with MG.

Cardiac investigation in patients with MG with unexplained fatigue should comprise a physical examination including third tone, holosystolic murmurs, ankle swelling, or lung rales; laboratory findings including brain natriuretic peptide (BNP) [8-10] and cardiac troponin [4]; 6-minute walk distance [13]; and echocardiography along with ECG. Diastolic impairment also affects the exercise intolerance and it is more likely to cause atrial fibrillation. $E / e^{\prime}$ in particular closely correlates with left ventricular filling pressure. Several prominent validation studies have confirmed this correlation and noted that the prediction of normal or abnormal filling pressure is most reliable when the ratio is $<8$ or $>15$, respectively $[14,15]$. In our study, the $E / e^{\prime}$ values were relatively high (mean: $10.46 \pm 3.24$ ) in patients in MG. There is a possibility that diastolic function was reduced in patients with MG. The diameter of the left atrium tends to be larger in patients with ECG abnormality; however, the combined measurement of BNP or N-terminal pro-BNP levels requires validation [8-10]. The mechanism underlying the high $E / e^{\prime}$ could be explained, at least partly, by the disturbance of calcium handling related to ryanodine receptors [16], which are one of the targets of autoimmunity in patients with MG [1]. A large-scale, long-term study is needed for verification, while the mechanism and clinical significance of diastolic dysfunction also remain to be elucidated.

There are several limitations of the present study. First, not all the patients with MG underwent ECG and echocardiographic examinations at our hospital and a selection bias may exist. Therefore, further prospective large-scale study or the analysis of consecutive cases is warranted to validate ECG as a useful tool for the screening method of cardiac dysfunction in 
TABLE 2: Clinical characteristics of patients with myasthenia gravis.

\begin{tabular}{|c|c|c|c|}
\hline & With ECG abnormality $(n=33)$ & Without ECG abnormality $(n=25)$ & $p$ value \\
\hline Male, $n(\%)$ & $15(48.4)$ & $9(50.6)$ & 0.903 \\
\hline Age, mean $\pm S D, y$ & $60 \pm 5.2$ & $58 \pm 6.1$ & 0.67 \\
\hline Time from diagnosis, mean $\pm \mathrm{SD}, \mathrm{y}$ & $12 \pm 8.0$ & $11 \pm 7.1$ & 0.67 \\
\hline Thymoma, $n(\%)$ & $8(24.2)$ & $10(40)$ & 0.08 \\
\hline \multicolumn{4}{|l|}{ Comorbidities, $n$} \\
\hline Diabetes mellitus & 1 & 2 & \\
\hline Systemic lupus & 1 & 2 & \\
\hline Rheumatoid arthritis & 0 & 1 & \\
\hline Sjogren disease & 0 & 1 & \\
\hline Death, $n$ & 3 & 3 & 0.512 \\
\hline \multicolumn{4}{|l|}{ Cause of death, $n$} \\
\hline Malignancy & 1 & 2 & \\
\hline Heart failure & 1 & & \\
\hline Acute myocardial infarction & 1 & & \\
\hline Aplastic anemia & & 1 & \\
\hline \multicolumn{4}{|l|}{ Type of treatment, $n(\%)$} \\
\hline Steroid & $19(55.4)$ & $10(40.0)$ & \\
\hline Cholinesterase inhibitor & $14(34.5)$ & $7(28.0)$ & \\
\hline Immunosuppressant & $12(34.2)$ & $11(44.0)$ & \\
\hline
\end{tabular}

ECG: electrocardiography; LV: left ventricle.

TABLE 3: Echocardiographic parameters of patients with myasthenia gravis.

\begin{tabular}{lccc}
\hline & With ECG abnormality $(n=33)$ & Without ECG abnormality $(n=25)$ & $p$ value \\
\hline Ejection fraction (\%) & $61.2 \pm 15.1$ & $66.1 \pm 4.8$ & 0.16 \\
LV end-diastolic dimension $(\mathrm{mm})$ & $44.7 \pm 5.8$ & $47.6 \pm 7.3$ & 0.14 \\
LV end-systolic dimension $(\mathrm{mm})$ & $31.8 \pm 9.5$ & $28.4 \pm 4.3$ & 0.90 \\
Septal wall thickness $(\mathrm{mm})$ & $8.9 \pm 1.6$ & $8.7 \pm 2.2$ & 0.63 \\
E/e' & $11.2 \pm 3.2$ & $34.1 \pm 5.6$ & 0.03 \\
Left atrium diameter $(\mathrm{mm})$ & $36.5 \pm 6.6$ & 0 & 0.20 \\
Wall motion abnormality & 5 ; diffuse & & \\
\hline
\end{tabular}

LV: left ventricle.

TABLE 4: Data concerning the LV damage and ECG abnormality.

\begin{tabular}{lccc}
\hline & $\begin{array}{c}\text { With LV } \\
\text { damage }\end{array}$ & $\begin{array}{c}\text { Without LV } \\
\text { damage }\end{array}$ & $(n)$ \\
\hline $\begin{array}{l}\text { With ECG } \\
\text { abnormality }\end{array}$ & $\begin{array}{c}14(5 \text { with } \\
\text { reduced EF })\end{array}$ & 11 & 25 \\
$\begin{array}{l}\text { Without ECG } \\
\text { abnormality }\end{array}$ & $\begin{array}{c}6(0 \text { with } \\
\text { reduced EF })\end{array}$ & 27 & 33 \\
$(n)$ & 20 & 38 & 58 \\
\hline
\end{tabular}

all patients with MG. Second, coronary artery disease could not be excluded entirely in these patients with MG, even though regional abnormality of wall motion, consistent with coronary artery disease, was not found in the present study. Third, whether an exercise test provoked the reduction of EF was not verified in the present study. BNP (or N-terminal pro-BNP) levels and physical findings were inadequate to be acquired. However, due to the rarity of the MG with cardiac involvement, this retrospective study has a clinical insight for the detection of systolic or diastolic dysfunction in patients with MG.

\section{Conclusions}

ECG may aid as the first step for the further examination of cardiac damage in patients with MG.

\section{Conflict of Interests}

The authors report no relationships that could be construed as a conflict of interests.

\section{Authors' Contribution}

Takao Kato and Sayako Hirose conceived and designed the experiments. Takao Kato, Sayako Hirose, and Shogo Kumagai analyzed the data. Takao Kato wrote the paper. Akihiko 
Ozaki, Sadayuki Matsumoto, and Moriaki Inoko critically reviewed the paper.

\section{Acknowledgment}

This study was supported by the Tazuke Kofukai Medical Research Institute.

\section{References}

[1] H. Hofstad, O.-J. Ohm, S. J. Mork, and J. A. Aarli, "Heart disease in myasthenia gravis," Acta Neurologica Scandinavica, vol. 70, no. 3, pp. 176-184, 1984.

[2] S. Suzuki, K. Utsugisawa, H. Yoshikawa et al., "Autoimmune targets of heart and skeletal muscles in myasthenia gravis," Archives of Neurology, vol. 66, no. 11, pp. 1334-1338, 2009.

[3] M. J. L. de Jongste, H. J. G. H. Oosterhuis, and K. I. Lie, "Intractable ventricular tachycardia in a patient with giant cell myocarditis, thymoma and myasthenia gravis," International Journal of Cardiology, vol. 13, no. 3, pp. 374-378, 1986.

[4] S. Kumagai, T. Kato, A. Ozaki et al., "Letter to the editor: serial measurements of cardiac troponin $i$ in patients with myasthenia gravis-related cardiomyopathy," International Journal of Cardiology, vol. 168, no. 2, pp. e79-e80, 2013.

[5] E. Venturini, A. Pellegrinetti, C. Marabotti, L. Magni, and R. Testa, "Myasthenia gravis and heart failure: is ivabradine a useful drug?" International Journal of Cardiology, vol. 174, no. 1, pp. e4-e6, 2014.

[6] R. J. Prineas, R. S. Crow, and H. Blackburn, The Minnesota Code Manual of Electrocardiographic. Findings: Standards and Procedures for Measurement and Classification, John Wright, PSG, Boston, Mass, USA, 1982.

[7] J. A. Kors, R. S. Crow, P. J. Hannan, P. M. Rautaharju, and A. R. Folsom, "Comparison of computer-assigned Minnesota codes with the visual standard method for new coronary heart disease events," American Journal of Epidemiology, vol. 151, no. 8, pp. 790-797, 2000.

[8] Japanese Circulation Society, "Guidelines for the clinical application of echocardiography," Circulation Journal, vol. 69, pp. 1409-1445, 2005.

[9] C. W. Yancy, M. Jessup, B. Bozkurt et al., "2013 ACCF/AHA guideline for the management of heart failure: a report of the American College of Cardiology Foundation/American Heart Association Task Force on Practice Guidelines," Journal of the American College of Cardiology, vol. 62, no. 16, pp. e147-e239, 2013.

[10] S. F. Nagueh, C. P. Appleton, T. C. Gillebert et al., "Recommendations for the evaluation of left ventricular diastolic function by echocardiography," Journal of the American Society of Echocardiography, vol. 22, no. 2, pp. 107-133, 2009.

[11] B.-Y. Xu, R. Pirskanen, and A. Kari Lefvert, "Antibodies against $\beta 1$ and $\beta 2$ adrenergic receptors in myasthenia gravis," Journal of Neuroimmunology, vol. 91, no. 1-2, pp. 82-88, 1998.

[12] T. C. Gibson, "The heart in myasthenia gravis," American Heart Journal, vol. 90, no. 3, pp. 389-396, 1975.

[13] L. Ingle, J. G. Cleland, and A. L. Clark, "The long-term prognostic significance of 6-minute walk test distance in patients with chronic heart failure," BioMed Research International, vol. 2014, Article ID 505969, 7 pages, 2014.

[14] S. R. Ommen, R. A. Nishimura, C. P. Appleton et al., "Clinical utility of Doppler echocardiography and tissue Doppler imaging in the estimation of left ventricular filling pressures: a comparative simultaneous Doppler-catheterization study," Circulation, vol. 102, no. 15, pp. 1788-1794, 2000.

[15] S. F. Nagueh, I. Mikati, H. A. Kopelen, K. J. Middleton, M. A. Quiñones, and W. A. Zoghbi, "Doppler estimation of left ventricular filling pressure in sinus tachycardia. A new application of tissue Doppler imaging," Circulation, vol. 98, no. 16, pp. 1644-1650, 1998.

[16] V. A. Lacombe, S. Viatchenko-Karpinski, D. Terentyev et al., "Mechanisms of impaired calcium handling underlying subclinical diastolic dysfunction in diabetes," The American Journal of Physiology-Regulatory Integrative and Comparative Physiology, vol. 293, no. 5, pp. R1787-R1797, 2007. 


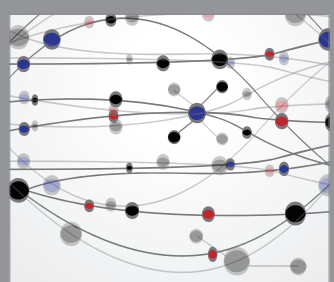

The Scientific World Journal
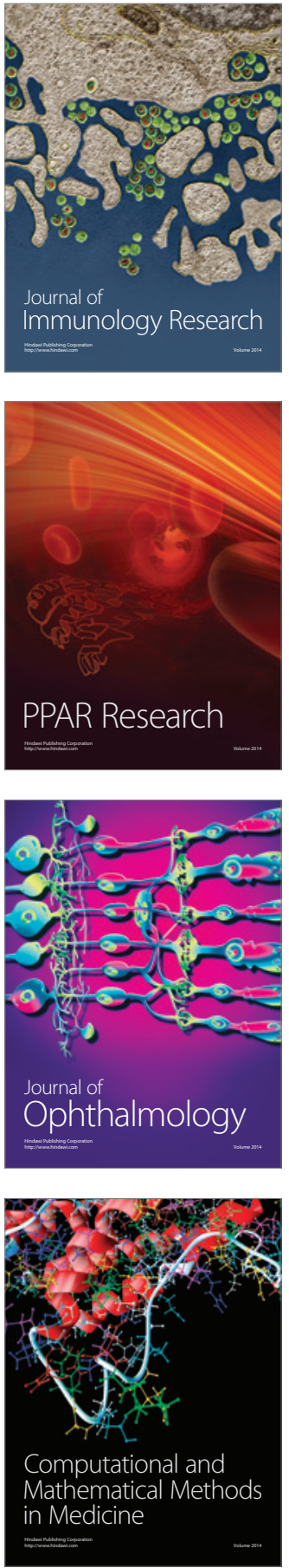

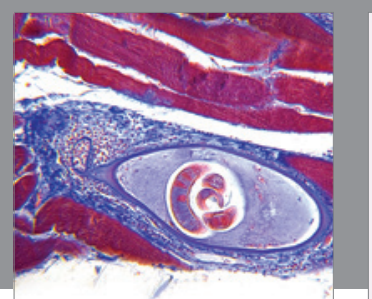

Gastroenterology Research and Practice

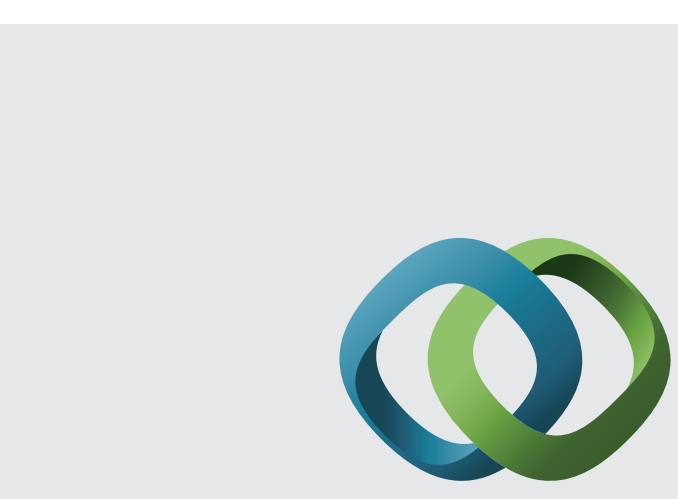

\section{Hindawi}

Submit your manuscripts at

http://www.hindawi.com



Disease Markers
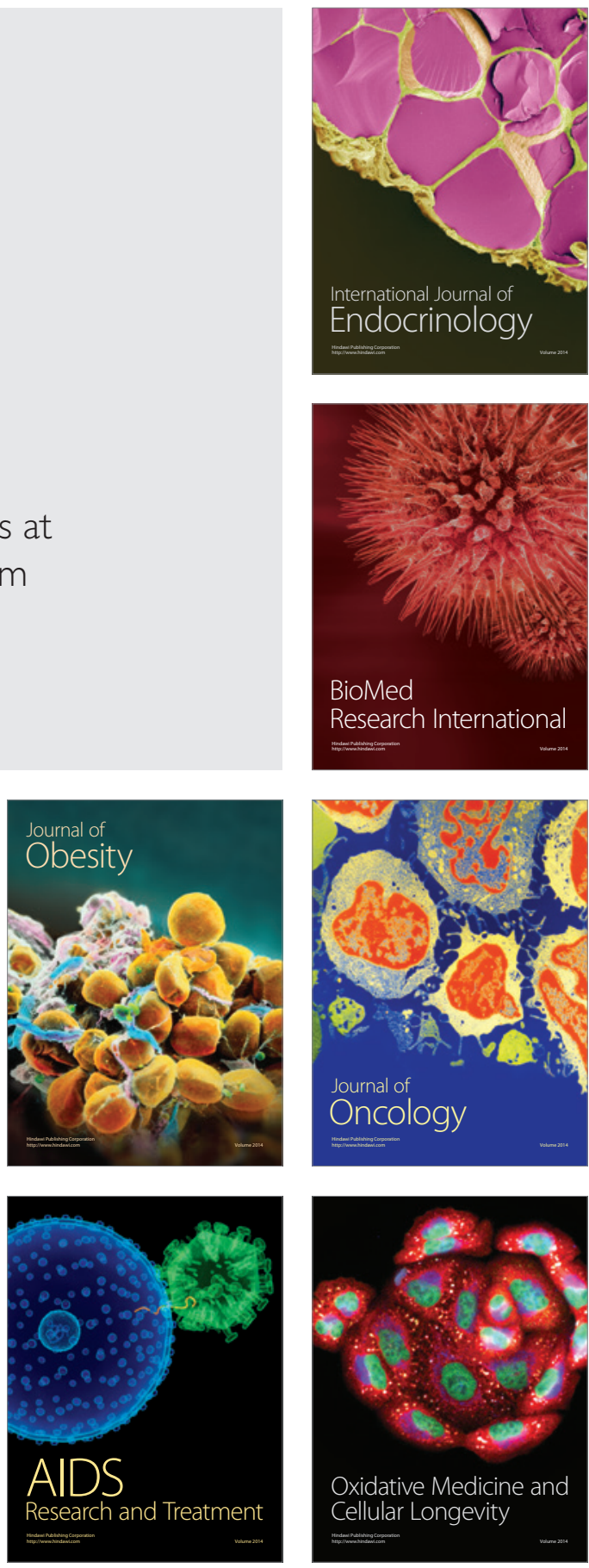